\title{
P I6-36. Functional genomic immune profile of SIV DNA vaccines delivered by electroporation and combined with molecular
} adjuvants

\author{
JD Boyer*1, J Yin1 ${ }^{1}$, A Dai ${ }^{1}$, S Belisle ${ }^{2}$, L Hirao ${ }^{1}$, R Palermo², D Hokey¹, J Yan ${ }^{1}$ \\ and D Weiner ${ }^{1}$
}

Address: ${ }^{1}$ Pathology, UPenn, Philadelphia, PA, USA and ${ }^{2}$ University of Washington, Seattle, WA, USA

* Corresponding author

from AIDS Vaccine 2009

Paris, France. 19-22 October 2009

Published: 22 October 2009

Retrovirology 2009, 6(Suppl 3):P265 doi:10.1 186/1742-4690-6-S3-P265

This abstract is available from: http://www.retrovirology.com/content/6/S3/P265

(C) 2009 Boyer et al; licensee BioMed Central Ltd.

\section{Background}

There are a number of challenges in the development of an effective vaccine for HIV, including stimulation of potent and broad cellular and humoral immune response that can cross protect between strains or clades. We have studied the cellular immune response, using genomics, of a DNA based vaccine that resulted in potent and broad cellular immune responses that suppressed SIV251 replication.

\section{Methods}

Rhesus macaques were immunized 4 times with $1.5 \mathrm{mg}$ of optimized SIVmac239 DNA vaccines, delivered by electroporation. Two groups of macaques received, along with vaccine, molecular adjuvants RANTES or IL-12. Macaques were challenged by the interrectal route, with high dose, unmatched SIVmac251. PBMCs were isolated and assessed by standard ELISPOT and proliferation methods for vaccine specific responses. PBMCs were also stimulated with vaccine antigens and RNA was isolated for functional genomics analysis.

\section{Results}

The most extraordinary result was that the RANTES molecule dampened the vaccine specific peripheral immune responses as assessed by antigen specific IFN-gamma production and proliferation but had a more significant impact on viral load compared to the DNA alone group. Using array analysis, the expression profiles of the differ- ent vaccine groups was compared. We found by ANOVA approximately 400 of 15,230 genes had altered expression in a vaccine specific manner compared to the control group. The gene expression levels were reflective of what was observed in biological assays. In addition, a functional genomics analysis of a second DNA vaccine study has now demonstrated using a rank-order analysis the same network of altered gene expression.

\section{Conclusion}

This is the first demonstration of an antigen specific response to a vaccine utilizing genomics. And this study provides a number of new immunological markers that may be relevant to vaccine analysis. This data that may be beneficial in providing guidance for future vaccinations regimens. 